\title{
RETOS E INCERTIDUMBRES EN LA PREDICCIÓN Y PREVENCIÓN DEL RIESGO SÍSMICO
}

\author{
Edgar Tapia Hernández ${ }^{(1)}$, Elizabeth A. Reddy ${ }^{(2)}$ y Laura Josabeth Oros Avilés ${ }^{(3)}$
}

\begin{abstract}
RESUMEN
El artículo discute desde un punto multidisciplinario el estado actual de las predicciones sísmicas con sustento científico basados en precursores físicos, métodos estadísticos y los requisitos mínimos que deben considerarse para identificar métodos razonables; especialmente, los encaminados a la prevención sísmica. Sobre la cultura de la prevención se discuten cambios históricos relacionados con la población vulnerable y marginal, su progreso en relación al desarrollo científico, la evidencia antropológica sobre la eficacia de las predicciones y su influencia en la cultura de la prevención para enfrentar riesgos comunes, el sistema de alerta temprana de sismos y la influencia de las predicciones en las expectativas y decisiones de inversión
\end{abstract}

Palabras clave: sismo; predicción; prevención; antropología; sistema de alerta; expectativas de inversión

\section{CHALLENGES AND UNCERTAINTIES IN THE PREDICTION AND PREVENTION OF SEISMIC RISK}

\begin{abstract}
This paper discusses from a multidisciplinary view, the state of the art of seismic predictions related to science-based studies based on physical precursors, methods based on statistical patterns and the minimum requirements to be considered in order to identify reasonable methods; especially, those aimed at prevention of seismic disaster. Culture of prevention, historical changes related to vulnerable and marginal populations, progress in relation to scientific development, anthropological evidence on the effectiveness of the predictions and their influence on the culture prevention to face common risks, seismic alert system and decisions and investment expectations derived from the predictions are discussed.
\end{abstract}

Keywords: earthquake; prediction; prevention; anthropology; alert system; investment expectations

Recibido el 5 de agosto de 2015, Revisado el 17 de enero de 2016, Aprobado el 8 de mayo de 2017. Se aceptarán comentarios y/o discusiones hasta cinco meses después de su publicación.

${ }^{(1)}$ Departamento de Materiales, Universidad Autónoma Metropolitana Azcapotzalco, Av. San Pablo 180, Col. Reynosa Tamaulipas, 02200 México, DF, e-mail: etapiah@azc.uam.mx

(2) Department of Sociology and Anthropology. Bucknell University, 701 Moore Ave, Lewisburg, P.A. 17837, U.S.A. e-mail: ear026@bucknell.edu

${ }^{(3)}$ Posgrado en Ciencias Económicas, Universidad Autónoma Metropolitana, Av. San Pablo 180, Col. Reynosa Tamaulipas, 02200 México, DF, e-mail: laurajosabeth@hotmail.com 


\section{INTRODUCCIÓN}

El 20 de marzo del 2012 se presentó un sismo con una magnitud 7.4 en la escala de Richter con epicentro en las cercanías de Ometpec, Guerrero y Pinotepa Nacional, Oaxaca en la costa sur de México. Pese a su magnitud, no hubo daños de importancia en las estructuras de las principales ciudades, por lo que el evento hubiera perdido relevancia en los medios de comunicación, si no se hubieran presentado varias réplicas (entre 3.9 a los 6.0 grados en la escala de Richter), que a la opinión pública parecían inusuales. Coincidentemente, en esos días se presentaron otros sismos alrededor del mundo (USGS 2015): el 14 de marzo en Honshu, Japón de 6.9 grados; el 21 de marzo en Nueva Guinea de 6.6 grados; el 25 de marzo en Maule, Chile de 7.1 grados; el 11 de abril en Sumatra de 8.6 y 5.5 grados; en Michoacán, México de 6.5 grados; el 12 de abril en el Golfo de California de 6.9 grados, por mencionar algunos.

Este panorama, alentó y supuestamente confirmó la utilidad de métodos de predicción de sismos con un sustento empírico sin comprobación previa ni demostración científica basados en la posición de los planetas, presentimientos, cambio climático, fuerzas exteriores al planeta provenientes del sistema solar, sueños, periodicidad supuestamente identificada previamente por la cultura Maya (a propósito del supuesto fin del mundo el 21 de diciembre del 2012), entre otras.

La realidad es que pese a la insostenibilidad técnica de estas teorías, la incertidumbre relacionada con los sismos alimenta el interés público a través de medios formales e informales de comunicación; de manera que logran posicionarse aún en eventos técnicos especializados. Por ejemplo, Curiel (2010) que sugiere "una temporada de terremotos" entre los meses de octubre a diciembre basada en fuerzas gravitatorias provenientes del exterior del planeta, sin que esta teoría tenga algún sustento científico o alguna tendencia estadística de consideración.

Esas teorías divulgadas popularmente y sin sustento científico no están necesariamente en oposición a las prácticas adecuadas para la reducción del riesgo sísmico y han destacado como potenciales recursos para la prevención de desastres conforme a los últimos "Acuerdos sobre la Reducción del Riesgo de Desastres" de la Organización de Naciones Unidas (UNISDR 2015) y en diversas situaciones como en Italia a raíz del terremoto del 2009 en L'Aquila (Alexander 2014) y los documentos en la fundación de Protección Civil en México (CNR 1986). Sin embargo, la propagación de información incorrecta e inexacta sobre predicciones puede crear confusión y desconfianza y originar consecuencias peligrosas para la población en situación de riesgo y hasta tener influencia en la confianza de inversión en un país. De hecho, el desarrollo de la cultura de la prevención ha demostrado ser considerablemente compleja, debido a la falta de intervención relevante, sostenida y sensible a nivel local y al diseño de intervención participativa.

Por otra parte, varios países a través de las universidades y agencias gubernamentales han desarrollado investigaciones con sustento científico basadas en observaciones de precursores geoquímicos e hidrológicos como programas para la predicción de sismos durante las últimas décadas. Por ejemplo, en 1980 se formó el Consejo Nacional para la Evaluación de la Predicción Sísmica (National Earthquake Prediction Evaluation Council) de Estados Unidos, que asesora al Director del Instituto Geológico sobre los métodos de predicción sísmica y la investigación científica relacionada con ellos. De manera, que alrededor del mundo se han logrado acumular registros confiables de varios tipos de observaciones en agua y gases en la superficie y estratos subterráneos que podrían ser usados como indicadores de los sismos y su proceso de generación. La predicción sísmica no es sólo un tema de investigación, sino que es de considerable interés popular, de tal forma que la difusión pública podría exigir la corrección de malentendidos.

Este artículo pretende difundir la información disponible y representa un compilado de resultados de investigaciones e información estadística con sustento científico para la predicción de los sismos; 
especialmente, aquellos esfuerzos que están encaminados en favorecer o incentivar la cultura de prevención y reducir las posibles pérdidas. Asimismo, se identifican las estrategias que se utilizan en la actualidad para integrar la sensibilización de los riesgos sísmicos en la conciencia popular a través de la cultura de la prevención, que incluye, aunque no se limita a:

a. Leyes, normas y reglamentos que tienen en cuenta la prevención de desastres.

b. Divulgación del conocimiento común sobre desastres potenciales y su influencia en la vida diaria.

c. Desarrollo de herramientas técnicas para situaciones de emergencia.

d. Esfuerzos continuos en educación sobre temas de riesgos y conocimiento especializado en temas de interés popular, como la predicción.

e. Desarrollo de los planes y procesos en una situación de emergencia.

f. El mantenimiento de canales de comunicación entre los diferentes actores involucrados en una situación de emergencia.

Así, el artículo pretende contribuir a una reforma cultural que proporcione beneficios significativos para la gestión de riesgos mediante el uso correcto de herramientas y, en general, la divulgación y aplicación de información precisa sobre la influencia de los terremotos en la vida cotidiana para maximizar su utilidad y minimizar los efectos negativos o perjudiciales de la información inexacta que circula actualmente.

\section{PRECURSORES BASADOS EN FENÓMENOS FÍSICOS}

\section{Medición de gases}

El 15 de noviembre de 1994 se presentó un temblor de magnitud 7.1 en la escala de Richter entre la isla de Luzón y Mindoro en Las Filipinas a 48 kilómetros al sur del volcán Taal, donde coincidentemente se había estado llevando a cabo una investigación sobre la emisión de gases del cráter (entre junio de 1993 hasta noviembre de 1996). El estudio, a cargo del profesor Patrick Richon del Departamento del Monitoreo del Medio Ambiente de Francia y otros colaboradores de Institutos y Universidades francesas, reportó un incremento inusual en las mediciones de un gas llamado Radón registrado veintidós días antes del sismo.

El Radón es el gas terrestre más estudiado como precursor de un evento sísmico y se trata del único gas noble radioactivo que existe en la naturaleza (incoloro, inodoro e insípido que en su forma sólida es de color rojizo). Existen tres isótopos del gas Radón: ${ }^{222} R n\left(T_{1 / 2}=3.825\right.$ días $),{ }^{220} R n\left(T_{1 / 2}=54.5 \mathrm{seg}\right)$ y ${ }^{219} R n$ $\left(T_{1 / 2}=3.92 \mathrm{seg}\right)$, de los cuales el más estudiado es el ${ }^{222} R n$ debido a su mayor tiempo de vida y mayor interés geoquímico. El isótopo ${ }^{222} R n$ pertenece a las series de decaimiento del isótopo de Uranio ${ }^{238} U$, que emite rayos $3 \alpha$, rayos $2 \beta$ y varios rayos $\gamma$ durante su desintegración en una condición de equilibrio. Así, que el monitoreo para el estudio de técnicas para la predicción de sismos se realiza midiendo los rayos $\alpha$ y/o rayos $\beta$.

Con base en los resultados obtenidos por Richon et al. (2003) y otros estudios similares, los científicos de la época tuvieron una congruente sospecha de que la anomalía en el gas Radón (principalmente el isótopo 222), concentrado en pozos de agua y en gases del suelo, podía estar asociada al aumento de esfuerzos de las placas tectónicas de la región lo que provocó finalmente el sismo de Mindoro en 1994.

Aunado a lo anterior, otros estudios habían identificado previamente variaciones geoquímicas antes de un sismo, como Hirotaka et al. en 1988 con mediciones del mismo gas Radón a 65 kilómetros del epicentro del sismo de Nagano en Japón de magnitud de 6.8 grados (Richon et al., 2003). Otro ejemplo, es el incremento en la concentración de Radón en mediciones del agua subterránea desde octubre de 1994 en la prefectura Hyogo, cuya magnitud máxima (diez veces más grande que la lectura inicial) se presentó el 8 
de enero; esto es, nueve días antes del famoso sismo de Kobe en Japón del 17 de enero de 1995 de 7.3 grados de magnitud (Igarashi et al., 1995). Más recientemente, se identificó el registro de altas concentraciones de gases, principalmente Radón $\left({ }^{222} \mathrm{Rn}\right)$, Helio $(\mathrm{He})$ y Dihidrógeno $\left(\mathrm{H}_{2}\right)$ cerca del epicentro del sismo de Wenchuan en China, que ocurrió el 12 de mayo del 2008 de magnitud de 8.0 grados (Zhou et al., 2010; Zheng et al., 2012).

Otros estudios, como Wakita et al. en 1991 identificaron una anomalía en las mediciones del gas Radón como un decremento (en lugar de un incremento como los otras investigaciones) que inició dos días antes del sismo de Japón de 1990 de magnitud de 6.0 grados (Igarashi y Wakita, 1995) cuyas mediciones se muestran en la figura 1.

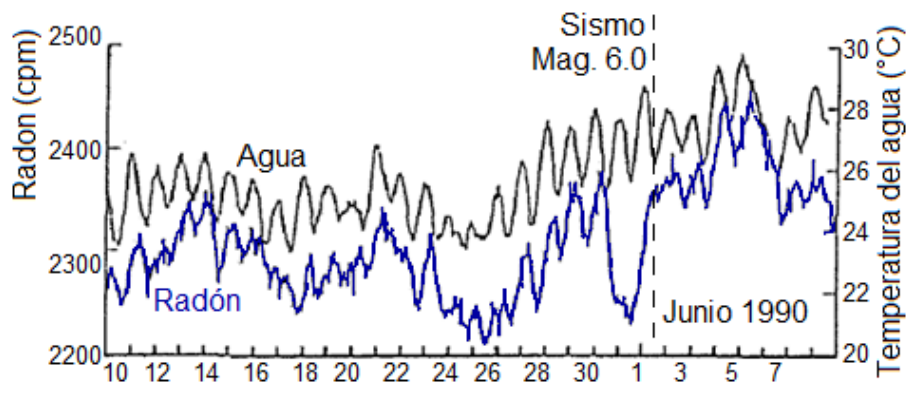

Figura 1. Comportamiento del gas Radón y temperatura del agua previo al sismo de Japón en 1990 (adaptada de Igarashi y Wakita 1995)

Así, la hipótesis de que la emisión del gas Radón ${ }^{222} R n$ está relacionada con un evento sísmico es simple: la compresión cercana a una falla cuyo movimiento es inminente produce emisiones de Radón ${ }^{222} \mathrm{Rn}$, como si el suelo fuera exprimido y, por lo tanto, una mayor emisión del gas anticiparía el terremoto. De hecho experimentalmente, Kato et al. en 1989 lograron establecer una correlación entre el incremento de la emanación del gas Radón ${ }^{222} R n$ ante un incremento de esfuerzos en experimentos de laboratorio en ejemplares de roca ensayados en compresión uniaxial (Igarashi y Wakita 1995).

A partir de esas y otras observaciones, se han realizado esfuerzos encaminados a medir las emisiones del gas Radón ${ }^{222} R n$ en todos los sitios posibles (Segovia et al., 1999; Omori et al., 2007; Richon et al., 2010) con el propósito de reducir el riesgo, principalmente en las poblaciones más vulnerables. Sin embargo, los resultados no han sido definitivos, con el paso del tiempo la teoría no ha sido comprobada, ya que los sismos se siguen presentando sin que se detecten las emisiones atípicas de gas en todos los casos (Richon et al., 2010). También se han reportado anomalías de la concentración de Radón ${ }^{222} R n$ que no son seguidas por sismos (Peña, 2003).

Asimismo, se han realizado predicciones basadas supuestamente en variaciones de la concentración del gas Radón con sitios, fecha y magnitudes equivocadas y que, posterior a algún evento se pretenden justificar. Como la predicción realizada por el técnico Giampaolo Giuliani previo al sismo de l'Aquila, Italia de abril de 2009 y que ahora es reconocido en internet como un supuesto previsor de sismos. De hecho, la predicción de Giuliani creó una poderosa reacción entre la población italiana, así como entre altos funcionarios estatales de Italia.

A partir de investigaciones cualitativas que consideran las mediciones de 150 estudios se ha hecho notar que el monitoreo de gases no es representativo de las concentraciones químicas del subsuelo a grandes profundidades, sino que las aguas termo-minerales han estado en contacto con grandes volúmenes de rocas de la corteza a varias profundidades, de modo que las mediciones son más representativas del entorno local 
del suelo y no del epicentro (Toutain y Baubron 1999; Peña 2003), ya que el deslizamiento de placas tectónicas que provoca un sismo se presenta a varios kilómetros de profundidad. De manera, que antes de considerar la variación de los gases como muestras para la identificación de precursores de terremotos, es necesario examinar su relación con perturbaciones ambientales como presión atmosférica, marea, temperatura ambiental, lluvia, presión barométrica, humedad del suelo o de la tierra, temperatura del viento, perturbaciones volcánicas (Segovia et al. 1999), etc. lo que demuestra la necesidad de una medición simultánea de parámetros externos durante la monitorización de gases y la creación de redes de estaciones.

En lo que respecta a la variación de otros gases, se han detectado anomalías en la concentración de Helio medidas como proporción de Argón (He/Ar) entre 1 a 3 meses antes del sismo del 14 de septiembre en 1984 al oeste de Nagano, Japón de magnitud de 6.8 grados. Las anomalías se detectaron en fumarolas de gas y en tres fuentes minerales en varios sitios con distancias al epicentro entre 9 a 95 kilómetros (Nagamine y Sugisaki, 1991).

Debido a que el proceso de generación de Hidrógeno en la corteza es complicado, es posible que el Hidrógeno pudiera funcionar eficientemente como indicador de la actividad de las fallas tectónicas. Kato et al. en 1989 demostraron experimentalmente que muestras de granito bajo carga uniaxial que la fractura de roca está relacionadas con la emanación de Hidrógeno $\mathrm{H}_{2}$; lo que fue confirmado por Stake et al. en 1985 que reportaron una emisión anómala de Hidrógeno $\mathrm{H}_{2}$ en cinco estaciones de medición antes del sismo del 26 de mayo de 1983 de magnitud 7.7 grados (Igarishi y Wakita, 1995).

También se ha medido la concentración de Carbono $\mathrm{C}^{-}$en manantiales minerales para la predicción de eventos sísmicos. Por ejemplo, Kaoizumi et al. en 1985 detectaron anomalías en la composición química (principalmente CI) a lo largo de las falla de Yoshioka en Japón (Peña, 2003).

\section{Observaciones geofísicas}

Otras investigaciones basadas en estudios cuantitativos físicos como la reflexión y refracción de ondas mecánicas, velocidad de las ondas sísmicas y métodos que se fundamentan en campos electromagnéticos, magnéticos o eléctricos y fenómenos radiactivos entre otros, también se han utilizado con el mismo fin. Por ejemplo, Derr en 1973 y Kamagowa et al. en 2005 registraron luminosidades relacionadas con un terremoto; Gokhberg et al. en 1982 y Nagao et al. en 2002 detectaron radiaciones electromagnéticas; Kondo en 1986 y Vershinin et al. en 1999 reportaron cambios en el campo eléctrico atmosférico; Malchanov y Hayakawa en 1998 y Liu et al. en 2000 registraron perturbaciones ionósferas (Omori et al., 2007).

En Turquía se estudió la composición de aguas termales a lo largo de 800 km de la falla de Anatolia durante tres años (2002 a 2004) para investigar la posible relación entre la actividad sísmica y las variaciones temporales en las características químicas e isótopos del agua. Aunque durante las mediciones no se presentaron sismos de magnitudes importantes, se observó una correlación entre las variaciones químicas y composiciones de los isótopos en algunos pozos con actividad sísmica de moderada magnitud (entre 3 y 5 grados) con epicentros cercanos a los sitios de los muestreos (Süer et al., 2008).

Algunas horas antes del terremoto del 26 de diciembre del 2004 en Sumatra, Indonesia de magnitud 9.1, se detectaron perturbaciones en la región F de la ionósfera (foF2 y hmF2) medidas desde Delhi, India mediante una sonda digital a distancia (Dutta et al., 2007).

Guo y Bin (2008) reportaron nubes inusuales 69 días antes del sismo de febrero de 2005 de 6 grados de magnitud en Zarand en Irán. Las espesas nubes extendidas a lo largo de la falla de Irán y temperaturas 
altas fueron determinadas a través de datos de sensores satelitales geoestacionarios. El estudio propone que las nubes pudieron ser modificadas por una emisión atípica de gases de la litósfera.

Desde otro enfoque, considerando un espacio de probabilidad $(F, \mathbb{P})$, siendo $F$ el conjunto de eventos, $\mathbb{P}$ el conjunto de probabilidades, $M$ el evento precursor y $N$ la ocurrencia de un sismo $(M, N \in \mathrm{F})$, donde la probabilidad de que ocurra el evento precursor siempre existe $\mathrm{P}(M)>0$ y la probabilidad de que tiemble en esa región geográfica también se cumple $\mathrm{P}(N)>0$. Entonces, la probabilidad condicional de $N$ dado $M$ se define con la ecuación 1.

$\mathrm{P}(M \mid N)=\frac{P(M \cap N)}{P(N)}$

$\mathrm{P}(M \mid N)$ se interpreta considerando que si $N$ se cumple, la porción condicional de $M$ se debe cumplir. Es decir, si el evento precursor identificado se presenta, la probabilidad condicional de que tiemble existe. El inconveniente de los métodos discutidos es que el espacio donde se puede presentar el precursor $M$ asociado a otros fenómenos naturales que no están relacionados con la actividad sísmica es grande. Igualmente, existe una alta probabilidad de que se presente un sismo sin que se presente el precursor $N$. Por esto, se han presentado los eventos precursores sin que posteriormente tiemble y también se han presentado sismos sin que se haya reportado el evento precursor $M$.

Así, aún hay mucho por hacer para establecer un patrón de referencia que se pueda comparar con las reacciones del medio ambiente: eventos asociados a estímulos potenciales en el laboratorio que permitan estudiar los fenómenos precursores de un terremoto real. Infortunadamente, la aleatoriedad natural y la actividad sísmica frecuente en ciertas regiones son usadas para hacer predicciones que pueden contar con una creencia absurda y peligrosa, que no pretende prevenir el riesgo. Por esta razón, la difusión de los métodos se debe realizar objetivamente, a partir de la formalidad de la metodología para establecer la ocurrencia, la cantidad de aciertos y las condiciones mediante las cuales fue comprobada.

\section{PRECURSORES BASADOS EN PATRONES ESTADÍSTICOS}

Existen otras metodologías basadas en correlaciones estadísticas, a partir de catálogos históricos de los sismos o métodos desarrollados a partir de modelos probabilísticos matemáticos. Estos métodos dependen de los datos recolectados con acelerómetros o de los datos recabados del análisis de evidencias físicas históricas de movimientos sísmicos y el análisis de evidencias sociales, a partir de la colaboración de antropólogos, geofísicos e historiadores de fuentes de la época prehispánica, la colonia y en el México independiente (García et al. 1988; García y Suárez 1996; Zuñiga et al. 1997).

De acuerdo a la teoría del rebote elástico, el proceso de generación de un sismo consiste en un periodo de acumulación de energía de deformación por el movimiento de las placas tectónicas y una liberación repentina que provoca el terremoto. Esa liberación de energía es precedida por un nuevo periodo de acumulación de energía hasta que se sobrepase la resistencia de las placas dando lugar a un nuevo sismo y así sucesivamente. Con este sustento, se han identificado áreas de contacto entre las placas que no han presentado actividad sísmica en un periodo de tiempo, conocidas como brechas sísmicas.

\section{Brecha sísmica}

Con base en la regularidad histórica, después del sismo de Michoacán, México de septiembre de 1985 de magnitud de 8.1 grados, se ha predicho un sismo que tendría epicentro en el sur de México en una zona conocida como la brecha sísmica de Guerrero. Esta zona se ubica en el Océano Pacífico en el área de 
subducción de la Placa de Cocos frente al Estado de Guerrero, desde el sur de Acapulco hasta Papanoa. La predicción se basa en que se identificó una alta probabilidad de que se presentara un sismo, a partir del momento sísmico acumulativo en la brecha de Guerrero en la que no se han detectado sismos desde 1911; es decir, se realizó una predicción en términos de ocurrencia con el propósito de estimular acciones que reduzcan el riesgo. Sin embargo, la predicción entre la población se difundió en términos de magnitud, donde se esperaba un sismo con magnitud igual o superior a los 8.1 grados.

Algunos investigadores han modelado estos procesos de ocurrencia de los sismos, a partir del catálogo de sismos históricos, recurriendo a modelos simplificados del proceso físico y modelos basados en la estadística bayesiana que hacen uso óptimo de la información disponible (Singh y Ordaz, 1994). De hecho, algunos de estos modelos han tenido efectos significativos en las políticas de Estado para la administración del riesgo sísmico en la población, expectativas de inversión, la difusión eficaz de información precisa, coberturas de riesgo, estimulación de acciones preventivas y en el desarrollo de herramientas concretas como el Sistema de Alerta Sísmica Mexicana.

Pese a esto, los años han pasado y el supuesto sismo predicho (desde antes de 1990) aún no ha ocurrido. Las explicaciones proponen que: a) la energía se sigue acumulando y tarde o temprano se va a liberar (aunque esto tampoco debería implicar que ocurrirá en un único sismo de gran magnitud) y b) otra teoría es que la liberación de la energía (el movimiento de las placas) se realizó mediante un terremoto silencioso. Esta teoría se fundamenta en la liberación de la energía, que provocaría un sismo, a través del desplazamiento de las placas durante varios días y que se han detectado a través del monitoreo de algunos sitios mediante estaciones de sistema de posicionamiento global (GPS). Por ejemplo, en la figura 2 se muestra la localización de estaciones GPS en suroeste de British Columbia, Canadá.

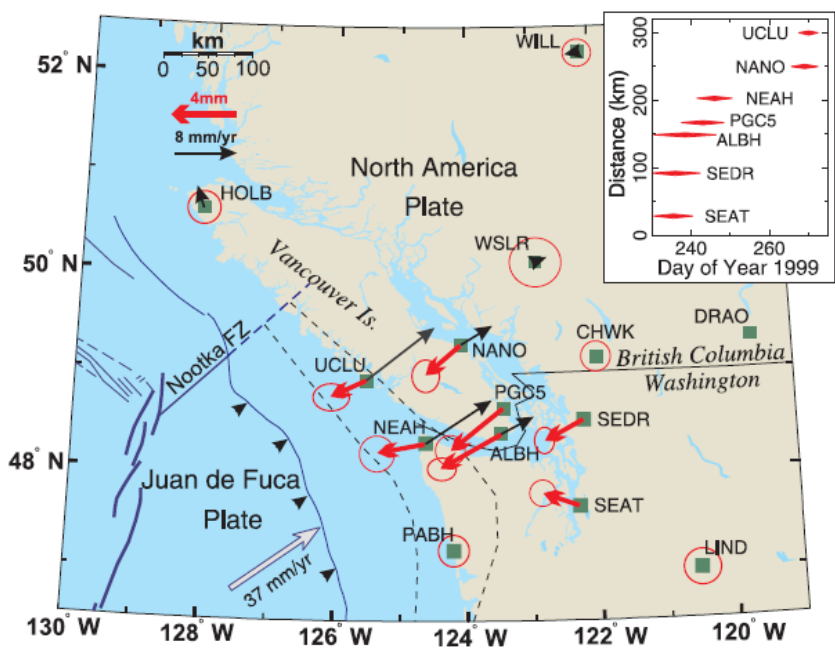

Figura 2. Movimiento de estaciones del sistema posicionamiento global (adaptada de Dragert et al., 2001)

Lo usual es que la posición de esas estaciones localizadas sobre la Placa de Norteamérica tiendan a moverse en dirección noreste (flechas negras) en un promedio de $8 \mathrm{~mm}$ por año (Dragert et al., 2001), debido al movimiento de subducción entre la placa Juan de Fuca y la placa Norteamericana. Sin embargo, en agosto de 1999, se presentó un desplazamiento de $4 \mathrm{~mm}$ en dirección suroeste (flechas rojas); esto es, las siete estaciones mostradas tuvieron un desplazamiento contrario al esperado que se desarrolló entre 6 y 15 días. Esto significa que durante ese periodo de tiempo se realizó una liberación de la energía equivalente a una magnitud de un sismo de 6.7 grados, pero sin que se registrara un sismo propiamente. 


\section{Probabilidad de ocurrencia}

En contraparte, la predicción acertada más célebre se registró previo al terremoto de Haicheng, China en 1975, donde la identificación de la brecha aunado a la observación de cambios en la elevación del suelo, los niveles de aguas freáticas, el comportamiento de los animales y pequeños temblores permitieron la evacuación de la población unas horas antes del sismo. Sin embargo, todo indica que la predicción se sustentó en una serie de coincidencias afortunadas y circunstanciales, en lugar de la aplicación de algún método verificado que estableciera una probabilidad de ocurrencia objetiva. Prueba de ello es que, los expertos chinos no han logrado predecir correctamente ningún gran evento desde entonces. De hecho, entre 1997 y 1999 se realizaron treinta predicciones equivocadas, lo que desestimó la credibilidad de los esfuerzos y obligó al gobierno chino a regular la publicación de las constantes alarmas para prevenir el pánico y las evacuaciones en masa (Saegusa, 1999).

Es valioso hacer notar que contrario a la creencia popular, un temblor de baja o moderada magnitud no libera la energía que evitaría un evento de magnitud importante; es decir, aunque los temblores pequeños ayudan a liberar la energía acumulada, no es posible que muchos temblores pequeños equivalgan a un temblor grande. Esto es, debido a que la escala de Richter establece magnitudes logarítmicamente, la liberación de energía no es equivalente entre los sismos: dos sismos de magnitud de 4 grados no equivalen a un sólo sismo de magnitud de 8 grados. De hecho, conforme al Programa de Riesgo Sísmico del Servicio Geológico de Estados Unidos, la energía acumulada en un sismo de magnitud 6 es equivalente unos 32 temblores de magnitud 5; unos 1,000 eventos de magnitud 4 ó unos 32,000 sismos de magnitud 3, etc. (USGS, 2015a).

De esta forma, aunque se identifique una cierta probabilidad de que se presente un evento sísmico con base en la identificación de cierta periodicidad, la realidad es que no existe una certeza fidedigna de que esto ocurra verdaderamente. La predicción en Parkfield, Estados Unidos es uno de los casos más conocidos (Mileti y Fitzpatrick 1992), donde se pronosticó un sismo originado en la falla de San Andrés, a partir de eventos previos registrados en 1857, 1881, 1901, 1922, 1934 y 1966 (Bakun et al., 1987). Así, el grupo de investigación identificó una brecha sísmica y un periodo de retorno aproximado de 22 años con una probabilidad de 90 a 95\% de que el sismo ocurriera entre 1985 y 1993 con una esperanza máxima en 1988. Sin embargo, el terremoto se presentó hasta septiembre del 2004, completamente alejado de la tendencia identificada.

\section{Frecuencia de un sismo}

Así, en el estudio de la sismicidad usualmente se pretenden determinar dos premisas básicas: a) la frecuencia con que pueden presentarse temblores de hasta una cierta magnitud en una región del mundo y b) la máxima magnitud sísmica que puede presentarse. Por lo anterior, la predicción de un sismo se define como la declaración de que un futuro terremoto pueda o no ocurrir en una región geográfica, un periodo de tiempo y una magnitud, con probabilidad (mayor a cero, pero menor a uno) de que el evento ocurra para advertir a la población sobre el riesgo sísmico.

De hecho, debido a que la búsqueda de precursores sísmicos que sirvan como un diagnóstico a corto plazo para la predicción de sismos no ha tenido éxito aún, se han centrado esfuerzos en la predicción operativa de terremotos como el medio para la recopilación y difusión de información sobre los riesgos sísmicos para ayudar a las comunidades a prepararse contra los terremotos destructivos.

Jordan et al. (2011) es un reporte de la Comisión Internacional para el Pronóstico de Terremotos para la Protección Civil que se formó en Italia después del sismo de L’Aquila en el 2009. La Comisión, que 
estuvo conformada por expertos de China, Grecia, Japón, Italia, Rusia y el Estados Unidos, realizó las siguientes recomendaciones al Departamento Italiano de Protección Civil (Jordan et al., 2011):

a. Apoyar la evolución científica del pronóstico probabilístico de terremotos y desarrollar los conocimientos necesarios para usar la información probabilista para efectos operativos.

b. Propiciar el flujo de datos de monitoreo sísmico y geodésico, a partir de laboratorios instrumentados que estudien el proceso de formación de los sismos.

c. Incentivar un programa de investigación básica sobre el conocimiento científico de terremotos y su previsibilidad.

d. Desarrollar modelos de pronóstico dependiente e independiente del tiempo para mejorar los mapas de peligrosidad sísmica a largo plazo orientados operacionalmente.

e. Mejorar la capacidad operativa de la predicción de réplicas y apoyar el desarrollo de métodos de predicción para cuantificar las variaciones de probabilidad a corto plazo.

f. Crear una comisión independiente de expertos para evaluar los métodos de predicción e interpretar sus resultados.

En la figura 3 se muestra por mes el número de sismos que se registraron entre los años 2007 y 2014 con una magnitud mayor a 5.0 en la escala de Richter en México. Con base en los resultados, disponibles en el catálogo de sismos registrados en el Servicio Sismológico Nacional (SSN 2015), no es posible identificar una tendencia en función de la época de lluvia o sequía, cercanía o lejanía de la Tierra del Sol, temperatura atmosférica, estaciones del año, ciclo lunar, etc.; de modo que la teoría de "temporada de sismos" difundida en redes sociales y otros medios de comunicación no tiene fundamento razonable.

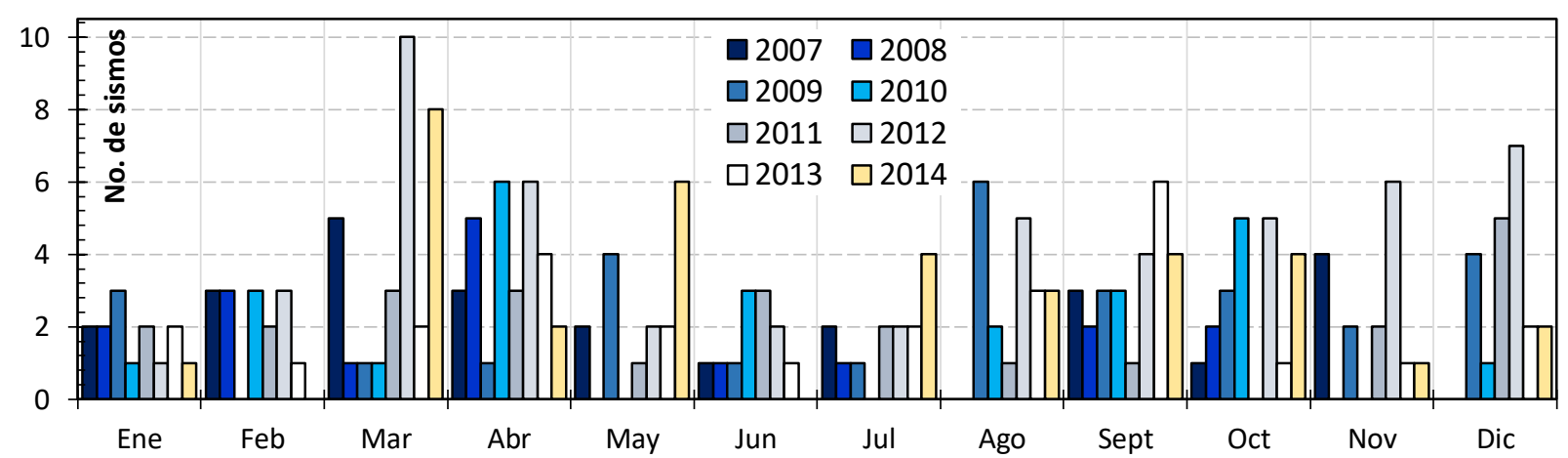

Figura 3. Número de sismos con magnitud $M \geq 5.0$ a lo largo del año en México

Aunado a lo anterior, no existe ningún fundamento para sospechar que el mes de septiembre esté asociado a algún precursor que promueva la ocurrencia de sismos, pese a que en ese mes haya acontecido el sismo de Michoacán del 19 de septiembre de 1985 de magnitud 8.1. Con el propósito ampliar esta discusión, en la figura 4 se muestran los sismos registrados en México con magnitud mayor a 3.0 en la escala de Richter durante el mes de marzo de los años 2010 a 2014. 


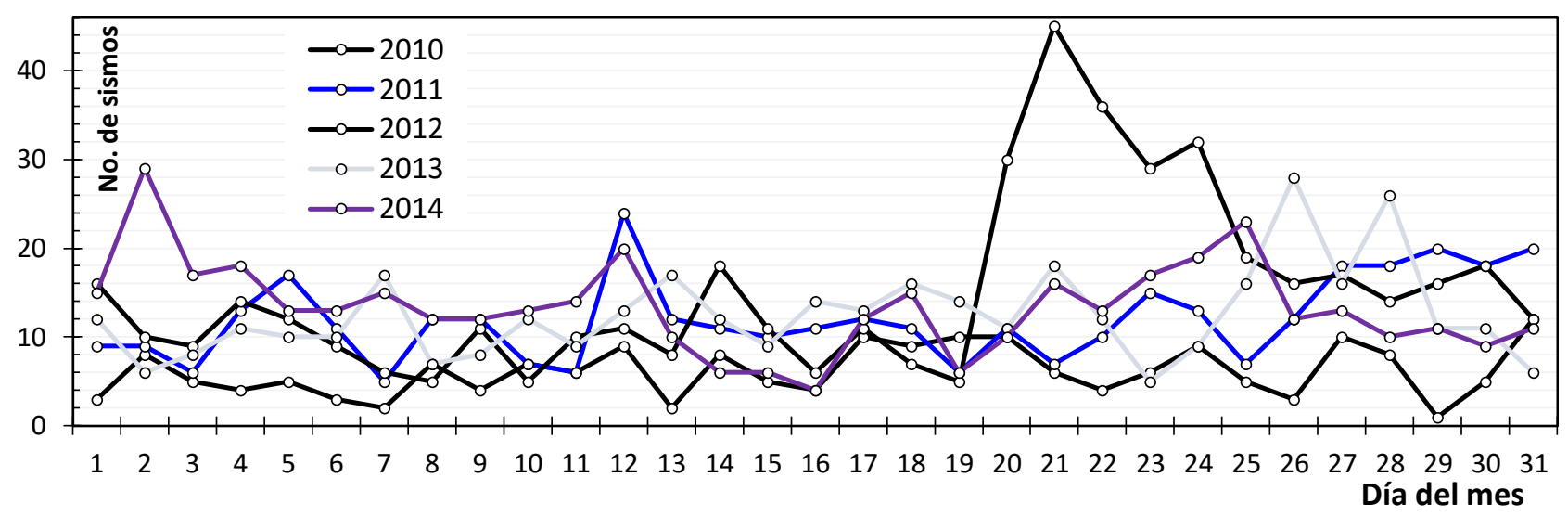

Figura 4. Número de sismos con magnitud $M \geq 3.0$ a lo largo de marzo en México

Es notoria la cantidad de eventos derivados del sismo del 20 de marzo del 2012 (comentado anteriormente); sin embargo, no es posible identificar una tendencia que haga sospechar que exista un periodo del año en el que algún precursor promueva la ocurrencia de sismos. Igualmente, se hace notar que es común que a lo largo de los días del año tiemble en México (Figs. 3 y 4), por lo que hacer una predicción de que cierto día ocurrirá un sismo es un hecho bastante probable. Pese a esto, argumentos similares son utilizados como método de comprobación de supuestas teorías sobre la predicción de sismos como Curiel (2010) y que son ampliamente difundidas en redes sociales, pese a que no pretenden reducir la vulnerabilidad de la población en riesgo.

\section{POBLACIONES EN RIESGO}

Desde la segunda mitad del siglo XX, los análisis de riesgos que se presentan en las poblaciones han puesto en evidencia que el desastre es un efecto social complejo (O'Keefe et al. 1976; García y Rojas 1994; Oliver-Smith 1996). Así, el desastre no es el resultado de un terremoto de gran magnitud, sino los efectos de ese terremoto y las condiciones de los sitios que afecta. La magnitud del riesgo, que las poblaciones pueden experimentar debido a los terremotos y otros peligros, es función de la probabilidad de peligro grave y también de su vulnerabilidad.

La vulnerabilidad tiene muchas caras y puede ser muy difícil de evaluar; por ejemplo, en los últimos "Acuerdos sobre la Reducción del Riesgo de Desastres" de Sendai de la Organización de Naciones Unidas (UNISDR 2015) se establecen riesgos sociales y estructurales como parte de la vulnerabilidad, destacando las formas en que la población vulnerable es desproporcionadamente afectada por un desastre. Los Acuerdos de Sendai definen vulnerabilidad, con referencia a los Acuerdos de Hyogo (UNISDR 2005), como "las condiciones determinadas por factores o proceso físicos, sociales, económicos y ambientales que incrementan la susceptibilidad de una comunidad al impacto de amenazas" (UNISDR 2015). Esto significa que factores como el conocimiento, ingresos, tipo de trabajo, red de apoyo social y las cualidades del entorno como viviendas y estructuras importantes, el acceso a servicios de electricidad, alcantarillado o carreteras y el agotamiento de recursos locales o la contaminación ambiental podrían ser efectos que se deben considerar para establecer la vulnerabilidad de una comunidad en desastre.

Las estrategias sugeridas por los Acuerdos de Sendai (UNISDR 2015) son muy variados e incluyen:

a. la comprensión del riesgo de desastres;

b. el fortalecimiento de la gobernabilidad para administrar el riesgo de desastres; 
c. la inversión en la reducción del riesgo de desastres para la resiliencia; y

d. mejoras en la preparación en caso de desastre para tener una respuesta, recuperación, rehabilitación y reconstrucción eficaz.

En este caso, la clave está basada en la comunicación de información precisa y útil entre los funcionarios y las comunidades de diversas vulnerabilidades. La integración de herramientas útiles de gestión de riesgos en la vida cotidiana de una población debe considerar diversas funciones que realizan investigadores, funcionarios locales, estatales, federales e internacionales, organismos no gubernamentales y la propia población en general.

Dado que muchas burocracias enfrentan retos de riesgos extremos (Dynes et al. 1990; Perrow 2008), el fomento de la cultura de la prevención es una forma de alentar los esfuerzos para disminuir con antelación los riesgos que plantean peligros potencialmente desastrosos y que implican daños, perdidas de inversión, compensaciones bancarias, cobertura de riesgo, etc.

\section{Cultura de Prevención}

Las ideas que los funcionarios y la población puedan desarrollar sobre la predicción de sismos (y sus limitaciones) son trascendentales para reducir el riesgo de la comunidad, ya que con ese conocimiento pueden tomar decisiones que afectan sus propias vidas, su seguridad y la de los demás. A menudo, la conciencia generalizada del riesgo se identifica como una deseable reducción del riesgo ante sismo (FEMA474, 2005); especialmente, si los miembros del población son representados mediante las posibles acciones que pueden tomar (Farley et al. 1993; Mileti y Fitzpatrick 1993) y por un fundamento sobre el cual desarrollar intervenciones adicionales (Gratton et al. 1987; Mileti y Fitzpatrick 1992). Este tipo de conciencia es más útil cuando la población afectada tiene una comprensión informada sobre cómo ocurren los sismos y sus efectos en las poblaciones; de manera que ese entendimiento puede ser usado en múltiples y creativas maneras para apoyar la repuesta ante una amenaza y la "resiliencia" del largo plazo (Barrios 2014). Los antropólogos definen culturas en términos de sistemas de práctica y el significado que engloban todos los aspectos de la vida humana, aunado a los aspectos técnicos y políticos, que usualmente son abordados. Teniendo esto en cuenta, la cultura de la prevención no puede estar limitada a la aplicación de los aspectos técnicos únicamente, sino que debe haber una interacción coherente entre la tecnología y los factores sociales para reducir la vulnerabilidad.

Una cultura de la prevención no depende necesariamente de la comprensión científica informada del riesgo. De hecho, esta comprensión no siempre es explícitamente necesaria para que se desarrollen acciones eficaces que enfrenten los riesgos. La evidencia antropológica sustancial indica que sistemas de creencias bien establecidas y penetrantes, ya sea científicamente informadas o desinformadas como algunos métodos de predicción sísmica, pueden ser probablemente más eficaces para enfrentar riesgos comunes. Las capacidades de adaptación y creatividad con base en tradiciones han representado un notable apoyo en el pasado (Torry 1978, Oliver-Smith 1994, Zaman 1994, Zaman y Haque 1991) y podría ofrecer lecciones de gran alcance para la mitigación de riesgos (Turton 1977; Oliver-Smith 1986).

En efecto, la utilidad potencial de las creencias locales no científicas ha sido apoyada por la ley, como ocurrió en L'Aquila, Italia. Donde después del terremoto de abril del 2009, se realizó un juicio contra expertos, en parte como una reacción social por haber demeritado las predicciones del técnico Giampaolo Giuliani (comentado anteriormente). Giuliani predijo con base en supuestas emanaciones de gas Radón que se aproximaba un gran terremoto, que finalmente ocurrió, y que los expertos aseguraron que era poco probable. Así, la opinión de los expertos disuadió a la población de seguir durmiendo fuera de sus casas, por lo que los expertos fueron declarados culpables de homicidio (aunque la sentencia ya fue anulada). Los marcos políticos incluyendo los Acuerdos de Sendai (UNISDR 2015) han destacado la forma explícita en 
que las prácticas informadas tradicionales son potencialmente útiles o incluso pueden ser tomados como modelos para el desarrollo de prácticas efectivas, aunque no tengan sustento científico.

Sin embargo, si la predicción de sismos no es necesariamente peligrosa en sí misma, los conceptos erróneos acerca de la predicción de los terremotos pueden interferir con las acciones que pueden potencialmente salvar vidas, ocasionando que sean intimidatorias u obstaculizando su aplicación. Aunque la investigación en ciencias sociales ha demostrado que el pánico es usualmente menos probable de lo esperado (Tierney 2003), la ansiedad relacionada con las predicciones de terremotos pueden afectar la actividad social y económica de una manera potencialmente significativa (Haas y Mileti 1976; Weisbecker et al. 1977; Echevarria et al. 1986). Por ejemplo, los costos por los daños de un terremoto, que son calculados usualmente en función de la expectativa de ganancia en un año económico, pueden generarse en ausencia de un terremoto real, a partir del movimiento de inversiones. Este tipo de impactos económicos en anticipación de un riesgo que puede no ocurrir se definen como una forma de "predicciones sísmicas psicológicas" (Olson et al. 1989).

Una cultura de la prevención efectiva debe trabajar fomentando las prácticas cotidianas en contra de predicciones sísmicas que promuevan la ansiedad ó una calma injustificada. De manera que, sin evitar los riesgos, reduzcan las vulnerabilidades que conducen al desastre. Esta cultura debe estar integrada en muchos sistemas y a todos los niveles.

Los responsables de la política y los expertos a menudo se refieren a otros aspectos de la cultura potencialmente eficaces: la cultura sísmica ó la cultura de la protección civil, que es una parte importante de la cultura mexicana de la prevención. Sus múltiples enfoques están implícitos en el desarrollo de la cultura de la prevención, aunque no necesariamente hacen hincapié a medidas a largo plazo. No obstante, los sistemas generalizados de valores existentes dirigidos a toda la población y las prácticas cotidianas para la mitigación de riesgos, la orientación sobre los desastres potenciales debería extenderse a la vida familiar e informar a la política de Estado también.

El desarrollo de la llamada "cultura sísmica" debería ser entonces una conjunción de conocimientos, creencias, habilidades, actitudes, conceptos relacionados con los terremotos, que se producen por la experiencia histórica (Karababa y Guthrie 2007; Mileti et al. 2002); es decir, un modo de ser, que podría haber existido en el México prehispánico, por ejemplo. De hecho, después del terremoto de Michoacán de 1985, expertos mexicanos hicieron notar que debía desarrollarse nuevamente (Carranza 1993).

Los esfuerzos en México por integrar la seguridad sísmica en la vida cotidiana se concibieron como una reforma cultural de Protección Civil desde su fundación. Esto pretende crear un ambiente de cambio para la introducción de nuevos valores, actitudes y conductas relacionadas con la protección civil. Además, pretende reafirmar las aptitudes y enseñar nuevas habilidades, elevar los niveles de aspiración y creatividad como incentivos para la conducta individual y colectiva y elaborar políticas y programas coherentes para ampliar el efecto de la protección civil en el proceso de cambio cultural (CNR 1986).

Estos esfuerzos por reformar las creencias y prácticas generalizadas sugieren que las medidas podrían redefinir las costumbres existentes o reformar las prácticas improductivas para motivar a la población a conducirse en forma más segura. Conforme a los estatutos de la fundación de Protección Civil en México, las medidas son apoyos esenciales y configuran procesos sociales y culturales de estructuración y desestructuración real y simbólica de la sociedad, intervienen en su organización y condicionan el modo de vida (CNR 1986). Entonces, con el paso del tiempo, la cultura de protección civil se convirtió en una prioridad mediante la Ley General de Protección Civil publicada en junio del 2012. De hecho, en noviembre del 2010 se publicó el decreto para crear el Instituto para la Seguridad de las Construcciones en el Distrito Federal. El Instituto es un organismo descentralizado de la Administración Pública, con la misión de 
verificar con ayuda de revisores los proyectos estructurales de nuevas edificaciones para garantizar el cumplimiento del Reglamento de Construcciones en la Ciudad de México y vigilar la aplicación de las normas y mediadas de seguridad en las edificaciones existentes.

La cultura sísmica y la cultura de protección, así como una cultura de prevención, implican que todos los actores se sensibilicen sobre los posibles riesgos, con el propósito de tomar decisiones que eviten efectos negativos. Un ejemplo de la difusión de prácticas adecuadas son los esfuerzos de la Universidad Nacional Autónoma de México (UNAM) por ofrecer cursos gratuitos sobre autoconstrucción (www.coursera.org/unam) y cada vez una mayor cantidad de cursos disponibles para estudiantes, arquitectos, ingenieros y directores responsables de obra como los que imparte el Centro de Actualización Profesional (CAPIT) del Colegio de Ingenieros Civiles de México (CICM), la Sociedad Mexicana de Ingeniería Sísmica (SMIS) y la Sociedad Mexicana de Ingeniería Estructural (SMIE) entre otras opciones, que pretenden actualizar el conocimiento de quienes deberán aplicar las normas modernas para el diseño sísmico.

En contraparte, los 2,457 municipios que existen en México están facultados para publicar su propio reglamento para el diseño sísmico; de manera que no existe un reglamento moderno que sea aplicable a todo el país. La situación de la normatividad local es muy variada: a) hay municipios que carecen absolutamente de directrices; b) en otros casos el reglamento establece requisitos de comportamiento, especifica cómo deben comportarse las estructuras sin definir cómo lograrlo; c) en otros casos se tratan de adaptaciones del Reglamento de Construcciones de la Ciudad de México y d) en muy pocos casos los reglamentos se basan en resultados de investigaciones sobre las condiciones locales. Esta variabilidad de criterios señala la falta de acción de las autoridades por reducir la vulnerabilidad sísmica.

De hecho, en el contexto de la protección civil, los esfuerzos que pretenden mitigar los riesgos se han criticado como una forma de descargar la responsabilidad de las autoridades por el bienestar general de la población (Macias 1999). Esta crítica podría aplicarse a algunos esfuerzos que fomentan la cultura de la prevención, particularmente, aquellas que enfatizan la importancia de la acción popular y el conocimiento sobre la regulación y el cumplimiento que sólo puede ser desarrollada por una autoridad gubernamental. Además, las intervenciones para cambiar prácticas locales pueden reiterar desigualdades perjudiciales de raza y clase (Lees 1980; Wolfe 1988; Lees y Bates 1990; Liebow y Wolfe 1993), por lo que deben realizarse a través de estrategias que inhiban o reduzcan posibles resentimientos sociales, pero sin dejar de reconocer la necesidad de reducir la vulnerabilidad. Así, la realidad es que no hay un conjunto de medidas claras para el desarrollo de una cultura de prevención y su aplicación tiene alta dependencia de las condiciones particulares de cada lugar. Pese a esto, hay mucho aún que aprender y desarrollar sobre los intentos de utilizar las herramientas científicas y técnicas para ayudar a las poblaciones en riesgo.

\section{EXPECTATIVAS DE INVERSIÓN ANTE UNA PREDICCIÓN}

En la teoría económica, la decisión de realizar una inversión debe analizarse con un enfoque intertemporal; es decir, la decisión está determinada por la relación entre las condiciones del pasado y el presente con las condiciones futuras, ya que el incentivo para hacer una inversión en el presente se basa en las posibilidades que existen de aumentar la producción en el futuro (Mankiw 2007). Uno de los primeros economistas en el siglo XX, que identificó los factores de incertidumbre que influyen en las decisiones de inversión fue John Maynard Keynes, un economista inglés (Keynes 1936). En especial, enfatizó que el estado de confianza de los empresarios en la previsión del rendimiento esperado es un factor determinante para decidir cuándo y dónde es rentable invertir. 
De acuerdo a Keynes (1936) existen dos tipos de expectativas en todo negocio: las expectativas de corto plazo y las de largo plazo. Las expectativas de corto plazo corresponden al precio que el productor espera obtener al inicio del proceso, por la venta de su producto terminado respecto al costo de producción. Mientras que las expectativas de largo plazo se refieren a lo que el empresario espera ganar por su inversión en forma de rendimiento futuro, mediante un aumento en los medios de producción; de manera, que las expectativas de corto plazo de los productores dependen en gran parte de las expectativas de largo plazo de los empresarios o inversionistas. Así, el estado de ambas expectativas son función de los pronósticos de las ganancias que puedan realizarse y del grado de confianza que los empresarios tengan en que efectivamente recibirán el rendimiento esperado. Donde la demanda de inversión del sector privado depende, entre otros factores como la tasa de interés, de las expectativas y del estado de confianza con el que los empresarios hagan sus previsiones.

Los métodos de predicción sísmica y sistemas de creencias bien establecidas y penetrantes, ya sea científicamente informadas o desinformadas, podrían modificar las expectativas de largo plazo de los empresarios, tanto locales como extranjeros, sobre el rendimiento futuro esperado y, por consiguiente, el gasto de la inversión.

Así, la posibilidad de un desastre natural en una cierta zona geográfica, que se considera vulnerable a través de una predicción con o sin fundamento científico, afecta el estado de confianza de los empresarios y puede disminuir la demanda de inversión. De hecho, esto ha sucedido en diferentes países que han enfrentado desastres naturales, donde la incertidumbre que genera el riesgo de que vuelva a ocurrir el fenómeno ocasiona que las pérdidas se amplifiquen. Uno de los ejemplos más relevantes del siglo XX fue el colapso inmobiliario que precedió a la Gran Depresión de 1929. En el otoño de 1926, se presentaron dos huracanes del Caribe que causaron daños devastadores en Florida, Estados Unidos dejando sin casas a miles de personas y un saldo de más de un millón de dólares de compensaciones bancarias. Esto provocó pérdidas en las inversiones realizadas y una gran incertidumbre en los posibles inversionistas, por lo que la demanda de inversión para la construcción de casas y hoteles cayó súbitamente en menos de un mes; aunado a un desesperado intento por vender y/o abandonar las inversiones realizadas previamente (Galbraith 1991).

Antes del desarrollo del sistema financiero, la decisión de inversión de un individuo o de una comunidad tenía un compromiso establecido mediante un contrato que era irrevocable. Sin embargo, en la actualidad, las inversiones se revalúan todos los días en la bolsa de valores, donde se promueve el traspaso de inversiones entre diferentes individuos. De ahí que muchas de estas nuevas formas de inversión se rigen más por el promedio de las expectativas de quienes participan en la bolsa de valores, que por las expectativas genuinas del empresario profesional basadas en un análisis racional. En otras palabras, en las valoraciones del mercado entran consideraciones de toda clase, como una predicción sísmica difundida en redes sociales y que de ninguna manera se relacionan con el rendimiento probable, sino más bien basados en una convención social (Keynes 1936). Entonces, en la bolsa de valores se decide invertir cuando hay una suposición de que la situación existente en los negocios continuará por tiempo indefinido, excepto cuando existan sospechas concretas de una modificación.

Cabe señalar, que en la bolsa de valores, la habilidad del inversionista y el especulador no se basan primordialmente en el rendimiento probable de la inversión, sino en la previsión de cambios en las bases convencionales. De modo que, la posibilidad de que ocurra o no un sismo en una cierta zona que se pregona vulnerable a través de una predicción influye en las expectativas de los inversionistas locales y extranjeros desincentivándolos a realizar operaciones en ese lugar e incentivan una decisión sobre la cobertura de riesgos, ya que previenen a) posibles pérdida, b) disminuciones en el rendimiento esperado y c) que el mercado devalúe la inversión. De hecho, basta con que un grupo de participantes del mercado esté convencido de la certeza de una predicción de un desastre natural, como un sismo, para que en el mercado de inversiones se propague el fenómeno de la psicología de masa. 
Keynes (1936) define la especulación financiera como el acto de prever la psicología del mercado y los rendimientos probables de los bienes. Cuando un empresario ordinario compra una inversión está poniendo sus esperanzas en un cambio favorable en las bases convencionales de valoración y no sólo en su rendimiento probable. Por lo que, las decisiones de inversión dependen del optimismo o pesimismo sobre el futuro y no sólo de una previsión matemática o económica.

Entonces, las decisiones de inversión son inestables ya que se basan en previsiones económicas y acontecimientos futuros influidos por la incertidumbre del mercado y convención social que pueden preverse con relativa seguridad. Este hecho explica que la inversión privada es considerada como la fuente principal de impulsos de las fluctuaciones macroeconómicas por la teoría económica y un factor que influye en el crecimiento de largo plazo de una economía (Mankiw 2007).

\section{SISTEMA DE ALERTA TEMPRANA}

La Sistema Alerta Sísmica Mexicana (SASMEX) inició como un sistema que permite advertir a los habitantes de la Ciudad de México que ha ocurrido un sismo en la costa del Estado de Guerrero en el área cercana a la brecha de Guerrero, comentada anteriormente. La Alerta Sísmica no predice la ocurrencia de un evento, sino alerta sobre un sismo que ya ha ocurrido; no constituye un método de predicción.

En la actualidad, el sistema consiste en una serie de 97 estaciones sísmicas ubicadas a lo largo de la costa sur y oeste de México (CIRES 2015), que al registrarse un sismo de una magnitud importante envía una señal de radio para advertir a la población. Es necesario hacer mención que el sistema funciona gracias a que la Ciudad de México se encuentra alrededor de $320 \mathrm{~km}$ de la costa (donde se presentan los epicentros del área de subducción entre la Placa de Cocos y la Placa Norteamericana) y a que la propagación de las ondas sísmicas en los estratos del suelo es de alrededor de $8 \mathrm{~km} / \mathrm{seg}$. Esto permite advertir a la población al menos 40 segundos antes de la llegada de un sismo, debido a que las ondas de radio viajan mucho más rápido que las ondas sísmicas.

El crecimiento de la red del sistema ha permitido incrementar los centros de población alertados como Oaxaca, Acapulco, Chilpancingo, Puebla y Morelia, las cuales tienen diferentes condiciones que la Ciudad de México y, por lo tanto, tienen intervalos de alerta más cortos; por ejemplo, en la Ciudad de Oaxaca es únicamente de diez segundos.

\section{Éxito y fracaso del sistema}

SASMEX se encuentra en operación desde 1991 (CIRES, 2015) con un éxito palpable en la sociedad. Actualmente, cuenta con el apoyo de estaciones de radio y televisoras locales para su difusión; así como repetidoras instaladas en edificios gubernamentales, agencias de repuesta emergencia, servicios de trasporte público, empresas privadas, hogares, edificios multifamiliares y escuelas, bocinas y, sin integración oficial, en los apps de teléfonos inteligentes personales. En México, la Secretaría de Educación Pública (SEP), el Gobierno del Distrito Federal (GDF) y el Gobierno Federal han distribuido más de 53,000 dispositivos pequeños de alerta y 150 dispositivos más grandes para escuelas solamente en la Ciudad de México (figura 5). Con ello, los niños en las escuelas pueden ser familiarizados con el sistema; de manera que, pueden desarrollarse simulacros organizados con facilidad (ver Arjonilla 1988 para la documentación de esa ventaja). Sin embargo, en realidad pocas personas fuera de los sistemas escolares y oficinas gubernamentales se han integrado en forma efectiva el sistema de alerta en sus vidas cotidianas. 

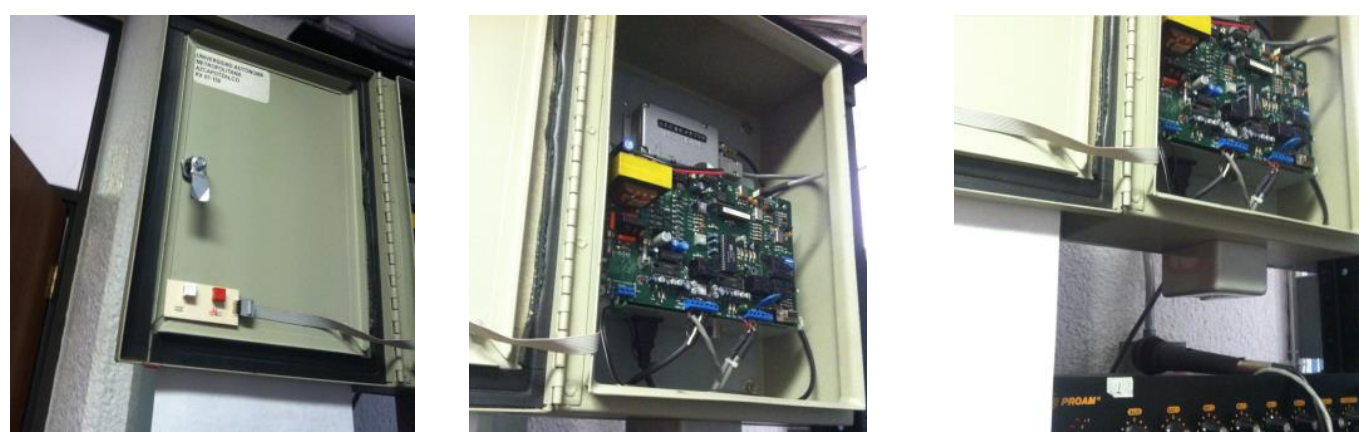

Figura 5. Dispositivo de alerta sísmica en una universidad pública

En la actualidad, hay una variedad de receptores comerciales disponibles, que en la mayoría de los casos, están fuera de la capacidad de compra del promedio de los mexicanos; aunque esto está cambiando gracias a las nuevas plataformas en radios, aplicaciones de teléfonos inteligentes y altavoces. De hecho, están en marcha esfuerzos para mejorar la tecnología de transmisión de datos desarrollados e instalados en teléfonos en México.

Los métodos de difusión que incorporan radiodifusión en lugares públicos y transmisiones de radio y televisión son más eficientes en sismos que ocurren durante el día, cuando la gente está en lugares donde los receptores están encendidos para emitir una señal notable de alerta con audio y luz. Esto es una limitación importante, puesto que en contraste, el sistema es poco eficiente en las noches cuando la gente está en sus hogares y están apagados los radios y televisores. Además, los apps de teléfonos inteligentes, pese a que son más económicos, no están oficialmente soportados (CIRES 2015) y tienen retrasos significativos.

Pese a que el SASMEX funciona correctamente (Suárez y García-Acosta 2014), su difusión es y ha sido un desafío. Aunque no hay investigaciones profundas sobre este tema, hay indicadores de que pocas personas en México comprenden cómo ocurren los terremotos o saben lo suficiente para evaluar eficientemente los sistemas de alerta sísmica. De hecho, sería idóneo que la respuesta de la población cuando escuchan la alerta sísmica varíe adecuándose a cada situación; por ejemplo, la respuesta de una persona debería ser diferente cuando están en un edificio alto, conduciendo un automóvil o en el aula de una escuela. Este objetivo requiere un conocimiento mínimo general, no sólo acerca del funcionamiento de la alerta y el intervalo de tiempo disponible antes de la llegada del sismo, sino sobre todo de prácticas de seguridad. De hecho, el ajuste de esos intervalos de tiempo requiere que los usuarios estén familiarizados con la sismicidad del área donde viven y que estén dispuestos a tomar acciones inmediatas al escuchar la alerta.

Los problemas que aún enfrentan las poblaciones mexicanas relacionadas con el SASMEX se resumen en lo siguiente:

a. Una porción de la población no sabe que existe el SASMEX de terremotos.

b. Una porción de la población sabe que existe el Sistema, pero no comprenden su funcionamiento, no pueden evaluar el SASMEX ni son capaces de compararlo con alertas fraudulentas.

c. Una porción de la población sabe que existe el Sistema, pero no pueden integrarlo en forma efectiva en su vida cotidiana.

Así, el reto para el SASMEX no es sólo técnico, sino que se trata de un desafío sobre la integración efectiva en los valores existentes y las prácticas cotidianas en las poblaciones. En otras palabras, la Cultura de Prevención no debe estar limitada a la difusión del conocimiento, sino que debe incluir e identificar los aspectos que aún están haciendo falta. Por ejemplo, una comunicación real entre los diferentes actores involucrados: los investigadores que desarrollaron y siguen refinando el Sistema de Alerta; organizaciones 
no gubernamentales como el Centro de Instrumentación y Registro Sísmico (CIRES); Protección Civil y funcionarios locales, estatales, federales e incluso a nivel internacional. Además, el Sistema debe incluir a miembros de la sociedad que no están en forma permanente en un sitio, sino que llevan vidas cotidianas móviles y no pueden ser fácilmente alertados; en esos y muchos otros casos, el uso de herramientas tecnológicas podría potencialmente salvarles la vida.

\section{CONCLUSIONES}

El artículo discute la factibilidad de la predicción y la cultura de la prevención de sismos desde un punto de vista multidisciplinario: antropológico, económico y de la ingeniería. El documento centra su atención en el estado actual de las predicciones sísmicas con sustento científico basados en precursores físicos, métodos estadísticos y los requisitos mínimos que deben considerarse para identificar métodos de predicción razonables; especialmente, los encaminados a la prevención sísmica. En la cultura de la prevención se discuten cambios históricos relacionados con la población vulnerable y marginal, su progreso en relación al desarrollo científico, la evidencia antropológica sobre la eficacia de las predicciones y su influencia en la cultura de la prevención para enfrentar riesgos comunes, el sistema de alerta temprana de sismos y la influencia de las predicciones en el grado de confianza y expectativas de inversión.

El artículo discute investigaciones que han identificado variaciones de mediciones que pueden ser consideradas como precursores de un sismo. Sin embargo, la gran mayoría de los reportes se basan en estudios de caso o ensayos retrospectivos que identifican un sismo como un evento que podría haber sido predicho con éxito, a partir del precursor que se identificó después de que ya se presentó el terremoto y que a posteriori ha sido difícil comprobar.

La predicción de los sismos tiene intrínsecamente una amplia cantidad de variables. Por ejemplo, las condiciones de cada región sísmica del mundo son demasiado particulares, lo que podría ocasionar que los precursores identificados para una cierta región geográfica no sean compatibles con las condiciones de otra parte del mundo. De manera general, una metodología propuesta para la identificación de precursores de un sismo debe considerar al menos los siguientes aspectos:

a. La metodología debe identificar un conjunto de sismos que se predijeron previamente y que se usaron para evaluar la efectividad del método propuesto.

b. Es necesario que el método limite su aplicación a la región del mundo para la cual ha sido probado. Dependiendo del método, la localización debe incluir información de la profundidad del movimiento y las coordenadas geográficas.

c. Además, debe especificar el intervalo de tiempo durante el que se realizaron las predicciones, caracterizado por una fecha de inicio y una fecha de culminación.

d. El método de predicción debe especificar un procedimiento definido para realizar una predicción acertada, lo que implica incluir ejemplos y pautas de la experimentación.

e. Finalmente, la propia metodología debe establecer el grado de incertidumbre asociada a la predicción; es decir, debe proporcionar una referencia del error. De hecho, sería racional que una predicción a corto plazo de un sismo esté asociado a un grado alto de incertidumbre.

A diferencia de otros fenómenos naturales como viento intenso provocado por huracanes, erupciones volcánicas o tormentas severas, los sismos aún no son predecibles con un simple monitoreo constante, sino que siguen siendo eventos naturales inadvertidos, recurrentes e inevitables. Pese a esto, el panorama no es desolador, puesto que se tiene absoluta certeza de las regiones del mundo que deben considerar medidas precautorias diarias de prevención sísmica. Esto debería guiar a las autoridades de todos los niveles, científicos, ingenieros de la práctica relacionados con el diseño y construcción de estructuras y a la población 
en general a dedicar esfuerzos para considerar, desarrollar y difundir medidas de seguridad para antes, durante y después de un posible sismo intenso.

De hecho, como se discute en el artículo, la evidencia antropológica sustancial indica que sistemas de creencias bien establecidas y penetrantes, ya sea científicamente informadas o desinformadas, pueden ser eficaces para enfrentar riesgos comunes y promover la cultura sísmica en términos de una cultura de protección y prevención. Al respecto, el desarrollo de la cultura de la prevención ha demostrado ser considerablemente compleja. Esto puede ser debido a la falta de intervención relevante, sostenida y sensible a nivel local y al diseño de intervención participativa. La investigación y la aplicación eficaz de los resultados debe ser un esfuerzo interdisciplinario, donde se aproveche la experiencia de los científicos sociales, ingenieros, economistas, entre otros. En este sentido, es necesario realizar investigaciones futuras adicionales que coadyuven a identificar:

a. Información incorrecta generalizada que sea clave con relación a la predicción sísmica.

b. La influencia de esa información incorrecta en el desarrollo de la cultura de prevención

c. Modos eficaces de comunicación científica que podrían apoyar localmente en forma apropiada la cultura de prevención.

Así, es probable que éxito de métodos de alerta como el sistema de alerta sísmica requieran que los usuarios estén familiarizados con la sismicidad del área donde viven y que estén dispuestos a tomar acciones inmediatas al escuchar la alerta. Es decir, el reto para el Sistema Alerta Sísmica Mexicana no debe estar limitada a la difusión del conocimiento técnico, sino que se trata de un desafío sobre la integración efectiva en los valores existentes y las prácticas cotidianas en las poblaciones; de manera que se debe incluir e identificar los aspectos efectivos para su integración en la vida cotidiana de la población. En su papel como conductor de cultura de prevención, la organización de Protección Civil puede ser un agente importante para apoyar ese proceso.

En el artículo se discute que los métodos de predicción sísmica y sistemas de creencias bien establecidas y penetrantes, ya sea científicamente informadas o desinformadas, pueden afectar las expectativas de inversión de largo plazo de los empresarios. De hecho, se demostró que la posibilidad de un desastre natural en una cierta zona geográfica ha afectado el estado de confianza de los empresarios y puede disminuir la demanda de inversión. En el artículo se discute que en las valoraciones del mercado entran consideraciones de toda clase, que de ninguna manera se relacionan con el rendimiento probable, sino más bien basados en una convención social.

\section{AGRADECIMIENTOS}

Los autores desean agradecer a un revisor anónimo por las sugerencias y aportaciones que mejoraron el manuscrito durante el proceso de arbitraje.

\section{REFERENCIAS}

Alexander D.E. (2014), "Communicating earthquake risk to the public: the trial of the 'L'Aquila Seven" Natural Hazards Vol. 72. pp.1159-1173. DOI:10.1007/s11069-014-1062-2

Arjonilla E. (1988), "Evaluación de la alerta sísmica para la Ciudad de México desde una perspectiva sociológica. Resultados en poblaciones escolares con y sin alerta" Internacional IDNDR - Conference on Early Warning Systems for Reduction of Natural Disasters. Chile. Abril. 
Bakun W.H., Breckenridge K.S., Bredehoeft J., Burford R.O., Ellsworth W.K., Johnston M.J.S., Jones L., Lindh A.G., Mortensen C., Mueller R.J., Poley C.M., Roeloffs E., Schulz S., Segall P. y Thatcher W. (1987), "Parkfield California, Earthquake Prediction Scenarios and Response Plans", Report 87-192 Geological Survey. Department of the Interior. Estados Unidos. Mayo.

Barrios, R.E. (2014), "Here, I'm not at ease: Anthropological perspectives on community resilience." Disasters, Vol. 38. Pp. 329-350. DOI:10.1111/disa.12044

Carranza J.A. (1993), "La Prevención y la cultura sísmica" Memorias, ler. Congreso Nacional de Universidades en Protección Civil. UNAM, diciembre. México

CIRES (2015), Sitio de internet del Centro de Instrumentación y Registro Sísmico, A.C. www.cires.mx. México. Fecha de consulta marzo 2015.

CNR (1986), "Bases para el establecimiento de una sistema nacional de protección civil. Protección Civil: México", Comisión Nacional de Reconstrucción. Distrito Federal.

Curiel G. (2010), "Teoría de las fuerzas gravitacionales", Memorias, XVIII Congreso Nacional de Ingeniería Sísmica, Aguascalientes, México.

Dragert H., Wang K.y James T.S. (2001), "A Silent Slip Event on the Deeper Cascadia Subduction Interface", Science Journal, Vol. 292. pp. 1525-1528. DOI:10.1126/science.1060152

Dutta H.N., Dabas R.S., Rupesh M.D., Kavita S. y Bhupender S. (2007), "Ionospheric perturbations over Delhi caused by the 26 December 2004 Sumatra earthquake", International Journal of Remote Sensing, Vol. 28, No. 13-14, pp. 3141-3151. DOI:10.1080/01431160601099590

Dynes R.R., Quarentelli E.L. y Wenger D., (1990), "Individual and Organizational Response to the 1985 Earthquake in Mexico City", Technical Report. Disaster Research Center, University of Delaware.

Echevarria J.A., Norton K.A. y Norton R.D. (1986). "The socioeconomic consequences of earthquake prediction". Journal of Earthquake Prediction Research, Vol. 4. Pp 175-193. ISSN 1002-1604

Farley J.E., Barlow H.D., Finkelstein M.S. y Riley L. (1993), "Earthquake hysteria, before and after: A survey and follow-up on public response to the browning forecast", International Journal of Mass Emergencies and Disasters Vol. 11 Issue 3. Pp. 305- 321. ISSN 0280-7270.

FEMA-474 (2005), "Promoting Seismic Safety: Guidance for Advocates". Federal Emergency Management Agency. Washington. Septiembre.

Galbraith, K. (1991), "Breve historia de la euforia financiera", Editorial Ariel, tercera edición, Barcelona, España.

García V., Hernández R., Molina I., Pérez A., Rojas J.M. y Sacristán C.T. (1988), “Cronología de los sismos en la cuenca del valle de México". Estudios Sobre Sismicidad en el Valle de México. Departamento Del Distrito Federal Secretaria General de Obras. México.

García V. y Suárez G. (1996), "Los Sismos en la Historia de México". Vol. 1.Universidad Nacional Autónoma de México Press. México.

García V. y Rojas T. (1994), "Los sismos como fenómeno social: una visión histórica", Macrosismos: Aspectos Físicos, Sociales, Económicos y Políticos, Ed. Centro de Investigaciones y Estudios Superiores en Antropología Social. México.

Gratton V.G., Their H.D., Arjonilla E. y Melgar R. (1987), "The recovery of schools from earthquake effects: Lessons from Mexico City", Disasters. Vol. 11, Issue 4. Pp. 310-316. DOI:10.1111/j.14677717.1987.tb00654.x 
Guo, G. y Bin W. (2008). "Cloud anomaly before Iran earthquake", International Journal of Remote Sensing, Vol. 29, No. 7. Pp. 1921-1928. DOI:10.1080/01431160701373762

Haas J.E. y Mileti D.S. (1976), "Social Effects of Earthquake Prediction", Ed. Institute of Behavioral Science, University of Colorado.

Igarashi G. y Wakita H. (1995), "Geochemical and Hydrological Observations for Earthquake Prediction in Japan”, Journal of Physics of the Earth, Vol. 43, No. 5, pp. 585-598. DOI:10.4294/jpe1952.43.585

Igarashi G, Saeki S., Takahata N., Sumikawa K., Tasaka S., Sasaki Y., Takahashi M. y Sano Y. (1995), "Ground-water Radon Anomaly Before the Kobe Earthquake in Japan", Science Journal, Vol. 269, No. 5220, pp. 60-61. DOI:10.1126/science.269.5220.60

Jordan T., Chen Y., Gasparini P., Madariaga R., Main I., Marzocchi W., Papadopoulos G., Sobolev G., Yamaoka K. y Zschau J., (2011) "Operational Earthquake Forecasting. State of Knowledge and Guidelines for Utilization", International Commission on Earthquake Forecasting for Civil Protection, Annals of Geophosics, Vol. 54, No. 4, mayo. DOI:10.4401/ag-5350

Karababa F.S. y Guthrie P.M. (2007), "Vulnerability reduction through local seismic culture”. Technology and Society Magazine, IEEE. Vol. 26. Pp 30-41. DOI:10.1109/ISTAS.2006.4375887

Keynes, J.M. (1936), “Teoría General de la ocupación, el interés y el dinero”, Fondo de Cultura Económica, cuarta edición.

Lees S.H. (1980), “The Hazards approach to development research: recommendations for Latin American drylands", Human Organization. Vol. 69. Pp 372-76. DOI:10.17730/humo.39.4.j221026444h54226

Lees S.H. y Bates D.G. (1990), "The ecology of cumulative change". The Ecosystem Approach in Anthropology, Ed. EF Moran, 1:247-78. Ann Arbor: University of Michigan Press.

Liebow E.B. y Wolfe A.K., (1993), “Communities at risk: communication and choice of environmental hazards" Environmental Health. Vol. 15 pp. 237-39. DOI:10.1186/1476-069X-9-67

Macías, J. M. (1999), "Desastres y Protección Civil: Problemas Sociales, Políticos y Organizacionales". Ed. CIESAS. México.

Mankiw, G. (2007), “Macroeconomía”, Antoni Bosch Editor, Cuarta edición, España.

Mileti, D.S. y Fitzpatrick C. (1992), "The causal sequence of risk communication in the Parkfield Earthquake Prediction Experiment", Risk Analysis, Vol. 12 Issue 3. Pp. 393-400. DOI:10.1111/j.1539-6924.1992.tb00691.x

Mileti D.S. y Fitzpatrick C. (1993), "The Great Earthquake Experiment: Risk Communication and Public Action", Ed. Westview Press. ISBN-13 978-0813383699.

Mileti D.S., Cress D.M. y Darlington J.D., (2002), "Earthquake culture and corporate action", Sociological Forum, Vol. 17. Pp. 161-180. DOI: 10.1023/A:1014549708645

Nagamine K. y Sugisaki R. (1991), "Co-seismic changes of subsurface gas compositions disclosed by an improved seismic-geochemical system”, Geophysical Research Letters, Vol. 18, Issue 12, pp. 22212224. DOI:10.1029/91GL02710

O’Keefe P., Westgate K. y Wisner B. (1976), "Taking the Naturalness Out of Natural Disasters.” Nature. Vol 260. DOI:10.1038/260566a0

Oliver-Smith A. (1986). "Natural Disasters and Culture Responses. Studies in Third World Societies", ERIC Institute of Education Sciences. Williamsburg, VA: Coll. William \& Mary.

Oliver-Smith A. (1994) “Peru's five hundred year earthquake: vulnerability in historical context” Disasters, 
Development, and Environment, Ed. A Varley, pp. 3-48. London: Wiley. ISBN-10 041591986x.

Oliver-Smith A. (1996), “Anthropological Research on Hazards and Disasters" Annual Review of Anthropology. Vol. 25. DOI:10.1146/annurev.anthro.25.1.303

Olson R.S., Podesta B. y Nigg J.M., (1989), "The Politics of Earthquake Prediction", Ed. Princeton University Press. Princeton, N.J. ISBN: 9780691608020.

Omori Y., Yasuoka Y., Nagahama H., Kawada Y., Ishikawa T., Tokonami S. y Shinogi M. (2007), "Anomalous radon emanation linked to pre-seismic electromagnetic phenomena", Natural Hazards Earth System Sciences, Vol. 7, pp. 629-635. https://hal.archives-ouvertes.fr/hal-00299460.

Peña P. (2003), "Cambios de radón en suelo y elementos químicos y trazas en agua subterránea asociados a actividad sísmica", Tesis doctoral, Universidad Autónoma Metropolitana, Abril.

Perrow C.B. (2008), "Complexity, Catastrophe, and Modularity”. Sociological Inquiry. Vol. 78 Issue 2. pp 162-173. DOI:10.1111/j.1475-682X.2008.00231.X

Richon P., Sabroux J., Punongbayan R. (2003), "Radon anomaly in the soil of Taal Volcano, the Philippines: A likely precursor of the M 7.1 Mindoro earthquake (1994)". Geophysical Research Letters. Vol. 30 (9), pp. 1481. DOI:10.1029/2003GL016902

Richon P., Klinger Y., Tapponnier P., Chen-Xia L., Vann Der Woerd J. y Perrier F. (2010), "Measuring radon flux across active faults: relevance of excavating and possibility of satellite discharges", Radiation Measurements, Elsevier, No. 45, pp. 211-218. DOI:10.1016/j.radmeas.2010.01.019

Saegusa A. (1999), "China clamps down on inaccurate warnings", Nature. International weekly journal of science, No. 397, 284. Enero. DOI: 10.1038/16760

Segovia N., Mena M., Peña P., Tamez E., Seidel J.K., Monnin M. y Valdés C. (1999), "Soil radón time series: surveys in seismic and volcanic areas", Radiation Measures. Vol. 31. Issues 1-6. Pp. 307-312. Junio. DOI:10.1016/S1350-4487(99)00142-0

Singh S. y Ordáz M. (1994), "Sismicidad y movimientos Fuertes en México: Una visión actual", Cuaderno de investigación 18. Centro Nacional de Desastres, Cenapred. México.

SSN (2015), Sitio web del Servicio Sismológico Nacional, México (www.ssn.unam.mx). Instituto de Geofísica, Universidad Nacional Autónoma de México.

Suárez G. y García-Acosta V., (2014), "The seismic alert system in Mexico City: an example of a successful Early Warning System (EWS)", UNISDR Scientific and Technical Advisory Group Case Studies.

Süer S., Gülec N., Mutly H., Hilton D.R., Cifter C. y Sayin M. (2008), "Geochemical Monitoring of Geothermal Waters (2002-2004) along the North Anatolian Fault Zone, Turkey: Spatial and Temporal Variations and Relationship to Seismic Activity", Pure and Applied Geophysics. Vol. 165, pp. 17-43. DOI:10.1007/s00024-007-0294-4

Tierney K. (2003), "Disaster Beliefs and Institutional Interests: Recycling Disaster Myths in the Aftermath of 9/11." Terrorism and Disaster: New Threats, New Ideas, Vol. 11, Research in Social Problems and Public Policy. Emerald Group Publishing pp. 33-51. ISBN: 978-0-76231-043-2

Torry W.I. (1978), "Natural disasters, social structure and change in traditional societies." Journal of Asian and African Studies. Vol 13. 167-183. DOI:10.1163/15685217-90007141

Toutain J.P. y Baubron J.C. (1999), "Gas geochemistry and seismotectonics: a review", Tectonophysics, Elsevier. Vol. 304, No. 1. Issues 1-2. Pp. 1-27. DOI:10.1016/S0040-1951(98)00295-9

Turton D. (1977), "Response to drought: the Mursi of southwestern Ethiopia." Disasters Vol. 1:275-87 DOI:10.1111/j.1467-7717.1977.tb00047.x 
UNISDR (2005), "Hyogo Framework for Action 2005-2015: Building the Resilience of Nations and Communities to Disasters", Organización de Naciones Unidas (ONU), Kobe, Hyogo, Japón. Enero.

UNISDR (2015), “Sendai Framework for Disaster Risk Reduction 2015-2030”, Organización de Naciones Unidas (ONU), Sendai, Japón. Marzo.

USGS (2015), "Historic World Earthquakes", Sitio web del Earthquake Hazard Program, United States Geological Survey, Department of the Interior. Abril. (http//earthquake.usgs.gov/earthquakes/world/historical.php).

USGS (2015a), "Earthquake Facts and Earthquake Fantasy", Sitio web del Earthquake Hazard Program, United States Geological Survey, Department of the Interior. Abril. (http://earthquake.usgs.gov/learn/topics/megaqk_facts_fantasy.php).

Weisbecker L.W., Stoneman W.C. y Ackerman S.E. (1977), "Earthquake Prediction, Uncertainty, and Policies for the Future: A technology assessment of earthquake prediction". Ed. Stanford Research Institute. Menlo Park, Calif.

Wolfe A.K. (1988) “Risk communication: Who's educating whom?” Pract. Anthropol. Vol. 10, pp. 13-14. DOI:10.17730/praa.10.3-4.gk353523755845p3

Zaman M.Q. (1994), "Ethnography of disasters: making sense of flood and erosion in Bangladesh.” Eastern Anthropologist. Vol. 47. Pp.129-155.

Zaman M.Q. y Haque C.E. (1991), "Coping with riverbank erosion hazard and displacement hazard in Bangladesh: survival strategies and adjustment." Disasters Vol. 13. Issue 4. Pp. 300-14. DOI:10.1111/j.1467-7717.1989.tb00724.x

Zheng G., Xu S., Liang S., Shi P. y Zhao J. (2012), “Gas emission from the Qingzhu River after the 2008 Wenchuan Earthquake Southwest China”, Chemical Geology, Elsevier. Vol. 339. Pp. 187-193. DOI:10.1016/j.chemgeo.2012.10.032

Zhou X., Du J., Chen Z., Cheng J., Tang Y., Yang L., Xie C., Cui Y., Liu L., Yi, Yang P. y Li Y. (2010), "Geochemistry of soil gas in the seismic fault zone produced by the Wenchuan Ms 8.0 earthquake, southwestern China", Geochemical Transactions Journal. Vol. 11:5. DOI:10.1186/1467-4866-11-5

Zúñiga R., Suárez G., Ordaz M. y García V. (1997), "Peligro Sísmico en Latinoamérica y el Caribe". Centro Internacional de Investigaciones para el Desarrollo. Proyecto 89-0190. Septiembre. Ottawa, Canadá. 